\title{
On the interaction of infrared radiation and nanocellular polymers: first experimental determination of the extinction coefficient
}

Victoria Bernardo ${ }^{* 1}$, Judith Martin-de Leon ${ }^{2}$, Javier Pinto ${ }^{2}$, Ulrich Schade ${ }^{3}$, Miguel Angel Rodriguez-Perez ${ }^{2}$

1. CellMat Technologies S.L., Paseo de Belen no9 A, 47011 Valladolid, Spain

2. Cellular Materials Laboratory (CellMat), Condensed Matter Physics Department, University of Valladolid, Campus Miguel Delibes, Paseo de Belén no7, 47011 Valladolid, Spain

3. Department of Locally Sensitive and Time-Resolved Spectroscopy, Helmholtz-Zentrum Berlin.

*Corresponding author: Victoria Bernardo (v.bernardo@cellmattechnologies.com)

\section{ABSTRACT}

Among the various mechanisms playing a role in the heat transfer through nanocellular polymers, the radiation contribution remains the most unknown, since there is a lack of experimental data about how infrared light interacts with such structures. In this work, we present the first experimental measurements of the transmittance in the infrared region of nanocellular polymers. Infrared transmittance spectra of a collection of polymethylmethacrylate (PMMA)-based micro- and nanocellular polymers with a constant density and a wide range of cell sizes (from $14 \mathrm{~nm}$ to $20 \mu \mathrm{m}$ ) were obtained and evaluated to calculate the extinction coefficient. Results show that, as expected from theoretical considerations, a reduction of the cell size increases the amount of infrared radiation transmitted, that is, the scattering is reduced as cell size reduces. Nanocellular polymers were proved to act as Rayleigh-like scattering points, showing the transmittance both an intense wavelength and cell size dependence. As a consequence, the extinction coefficient reduces in the nanoscale. From these data, it is possible to conclude that the scattering due to the cellular structure can be neglected for very small cell sizes (smaller than $200 \mathrm{~nm}$ ), but it must be considered for larger cell sizes. The obtained results were used to model the thermal conductivity including the radiation contribution, showing that at low relative densities and small cell sizes this heat transfer term becomes significant in nanocellular polymers.

\section{KEYWORDS}

Extinction coefficient; thermal conductivity; nanocellular polymers.

\section{INTRODUCTION}

Thermal insulation plays a major role in controlling the efficient use of energy in buildings since the energy used to keep a pleasant temperature in indoor spaces (more than $50 \%$ of the total energy spent in the sector [1]) could be reduced with improved insulation systems. Among the different solutions used for thermal insulation [2,3], cellular polymers have the main advantage of presenting excellent insulation properties, while being reasonably low-cost and easy to produce and install [4].

The parameter describing the thermal insulation ability of a given material is the thermal conductivity, $\lambda$. For cellular polymers, the thermal conductivity is governed by four mechanisms as shown in equation (1): conduction of heat through the solid polymer, $\lambda_{s}$, 
conduction of heat through the gas inside the cells, $\lambda_{g}$, convection of heat through the cells, $\lambda_{c}$, and radiation through the structure, $\lambda_{r}[4,5]$ :

$$
\lambda=\lambda_{s}+\lambda_{g}+\lambda_{r}+\lambda_{c}
$$

The radiation term accounts for over $20-30 \%$ of the total heat transfer in low-density cellular polymers, and it is probably the most challenging contribution to understand and quantify [5]. Over the years, several equations have been proposed to model the radiation term, such as those of Williams and Aldo [6] or Glicksman [7].

One unsolved question regarding the radiation contribution concerns the limits of the current models when the cell size is very small. According to the existing equations $[6,7]$, the radiation contribution decreases as the cell size decrease. However, when the cell size is no longer much larger than the infrared radiation wavelength, as many models hypothesize, the current approximations might not be still valid.

There is previous evidence to expect a different transfer of radiation in nanocellular polymers (i.e., cellular polymers characterized by nanometric cell sizes). For example, it has been recently proved that nanocellular polymers can be transparent to visible light when the cell size is small enough $[8,9]$. In particular, it is necessary that the cell size is smaller than $1 / 10$ of the light wavelength to obtain transparency. Taking into account that the wavelength of visible light is centered around $500 \mathrm{~nm}$ (green light), nanocellular polymers with cell sizes under 50 $\mathrm{nm}$ would become transparent, as already proved by Martin-de Leon and coworkers [9]. Also, at this scale, the scattering mechanism suffered by the light is Rayleigh-like, meaning that there is a strong dependency with the wavelength in the scattering process. As a consequence, nanocellular polymers present a bluish color [10]. Then, it is evident that cell size has something to say in the interaction with electromagnetic waves.

At room temperature, the maximum of the radiation emitted by a blackbody is centered in the infrared region, so to predict the radiation contribution in nanocellular polymers, it is necessary to understand how infrared radiation interacts with such structures.

When infrared radiation travels through a cellular structure, two main processes take place: absorption and scattering. The first one is determined by the amount of solid polymer (that is, the relative density) and the type of polymer, so the cell size does not affect this mechanism. However, the scattering phenomenon is strongly affected by the size of the scattering points (i.e., the cells). In aerogels, that present similar structural characteristics to nanocellular polymers, it was proved that there is a high transmittance in the infrared region in frequencies where the solid material does not have an absorption band [11], meaning that the scattering is reduced for nanometric cell sizes. In the work of Hrubesh et al. [12], the radiation contribution in aerogels was associated only with the absorption of the solid material, assuming that there is not scattering in the cells due to their reduced cell size [13].

Up to date, and as far as the authors know, there is not any experimental evidence of this effect in nanocellular polymers. In the 1D model proposed by Ferkl et al. [14], the radiation contribution decreased with the reduction in cell size in nanocellular polymers. In the 3D model of Wang and coworkers [15], the radiation contribution in nanocellular polymers was shown to be significant when density reduces, although the calculation of the radiative contribution is purely theoretical and was not experimentally validated. 
In this work, we have studied for the first time the interaction of infrared light with nanocellular polymers to investigate the contribution of radiation to the total thermal conductivity. The extinction coefficient of a collection of nanocellular polymers with variable cell size has been measured. According to the Rosseland equation [16-18], the radiation term can be calculated as expressed in equation (2):

$$
\lambda_{r}=\frac{16 n^{2} \sigma T^{3}}{3 K_{e, R}}
$$

Where $\sigma$ is the Stefan-Boltzman constant, $T$ is the temperature, $n$ is the refractive index, and $K_{e, R}$ is the Rosseland extinction coefficient. Thus, this parameter is essential to quantify the influence of the cell size in the radiation contribution. Our results show that, as expected from a theoretical perspective, the decrease of the cell size causes a drastic decrease of the extinction coefficient, and thus an increase of the radiation contribution.

\section{EXPERIMENTAL}

\subsection{Materials}

Table 1 summarizes the main structural characteristics of the collection of polymethylmethacrylate (PMMA)-based micro- and nanocellular polymers used in this work. All the materials were produced using a two-step gas dissolution foaming process and have similar relative densities. Details about the production process used to produce each sample can be found in the corresponding reference. The PMMA grade used as polymer matrix was, in all cases, ALTUGLAS ${ }^{\circledR} \mathrm{V} 825 \mathrm{~T}$ kindly supplied by Arkema. In the samples with MAM (poly(methyl methacrylate-poly(butyl acrylate)-poly(methyl methacrylate)), the amount of block copolymer was always low (smaller than $2 \mathrm{wt} \%$ ) [19] and the infrared spectrum was not modified due to the addition of MAM. The average 3D cell size of the samples was measured by SEM plus image analysis [20], while the relative density was determined by the Archimedes' principle after polishing the samples to remove the solid skin.

Table 1. Characteristics of the PMMA-based micro- and nanocellular polymers used in this work.

\begin{tabular}{|c|c|c|c|c|}
\hline Sample \# & Cell size & $\begin{array}{c}\text { Relative } \\
\text { Density }\end{array}$ & Material & Reference \\
\hline 1 & $14 \mathrm{~nm}$ & 0.42 & PMMA & {$[9]$} \\
\hline 2 & $24 \mathrm{~nm}$ & 0.43 & PMMA & {$[9]$} \\
\hline 3 & $120 \mathrm{~nm}$ & 0.37 & PMMA/MAM & {$[19]$} \\
\hline 4 & $225 \mathrm{~nm}$ & 0.42 & PMMA & {$[21]$} \\
\hline 5 & $700 \mathrm{~nm}$ & 0.41 & PMMA/MAM & {$[19]$} \\
\hline 6 & $800 \mathrm{~nm}$ & 0.43 & PMMA/MAM & {$[19]$} \\
\hline 7 & $2.5 \mu \mathrm{m}$ & 0.43 & PMMA & {$[22]$} \\
\hline 8 & $3.5 \mu \mathrm{m}$ & 0.43 & PMMA & {$[23]$} \\
\hline 9 & $16 \mu \mathrm{m}$ & 0.43 & PMMA & {$[24]$} \\
\hline 10 & $20 \mu \mathrm{m}$ & 0.41 & PMMA & {$[24]$} \\
\hline
\end{tabular}

Thin plane-parallel sheets were obtained from the foamed samples of Table $\mathbf{1}$ with a precision cutting machine (Model 1000 IsoMet). Homogenous and uniform samples of various 
thicknesses for each material in the range from 30 to $130 \mu \mathrm{m}$ were obtained for the transmittance measurements. The thickness of the samples is always higher than the wavelength of the infrared radiation used. All the samples were produced using the same method, so the surface quality of all the samples was comparable. The thickness of the samples was measured using a DMA7 dynamic mechanical analyzer (PerkinElmer) with an accuracy of 0.5 microns.

\subsection{Transmittance measurements}

The infrared transmittance of the samples was measured by using an IR microscope Nicolet iN10 MX (Thermo Scientific) located at the synchrotron BESSY II (Berlin). Infrared spectra were collected in a wavelength range from 2.5 to $12.5 \mu \mathrm{m}$ (800 to $4000 \mathrm{~cm}^{-1}$ in wavenumber). 128 scans were acquired. Before each measurement, the background signal was corrected. The spot size was $50 \times 50 \mu \mathrm{m}^{2}$, and every sample was measured at the least at two regions to assure the reproducibility of the measurements.

\subsection{Calculation of the extinction coefficient}

For thin homogeneous samples, the spectral extinction coefficient $K_{e, \lambda}$ can be obtained with Beer's law (equation (3)), where $T_{\lambda}$ is the transmittance at a given wavelength $\lambda$, and $L$ is the sample thickness. By means of a linear regression of the infrared transmittance spectra at various thicknesses, $K_{e, \lambda}$ can be calculated.

$$
K_{e, \lambda}=-\frac{\ln \left(T_{\lambda}\right)}{L}
$$

The Rosseland mean extinction coefficient, $K_{e, R}$, can be calculated from the spectral coefficient according to equation (4):

$$
\frac{1}{K_{e, R}}=\frac{\int_{0}^{\infty} \frac{1}{K_{e, \lambda}} \frac{\partial e_{b, \lambda}}{\partial T} d \lambda}{\int_{0}^{\infty} \frac{\partial e_{b, \lambda}}{\partial T} d \lambda}
$$

Where $e_{b, \lambda}$ is the spectral black body emissive power and $T$ is the temperature.

Due to the high density of the nanocellular samples used in this work, the absorption bands corresponding to the solid PMMA in the transmittance spectra are saturated even at very low thicknesses (see an example in Figure 1). Therefore, it is not possible, with these samples, to perform the previous analysis and give a global Rosseland extinction coefficient. However, as it was explained in the introduction, the interaction of the infrared light with cellular polymers can be divided into two terms: first, the absorption part (that is, the amount of radiation absorbed by the polymer, that depends on the density and the polymer nature), and the scattering contribution, which is mainly due to the presence of the cellular structure. Then, it is possible to define $K_{e, R}$ as a sum of these two contributions, as already proposed by Glicksman [7]: an absorption Rosseland term, $K_{e, R, a b s}$, and a scattering term $K_{e, R, s c a t t}$ (equation (5)):

$$
K_{e, R}=K_{e, R, a b s}+K_{e, R, s c a t t}
$$


The transmittance curve can be then divided into these two contributions: the absorption bands and the scattering baseline. Figure 1 also shows the scattering baseline once the absorption bands are removed. This baseline was calculated by selecting a collection of points out of the absorption bands and then performing a fit of those points. In the region of wavenumbers from $1900 \mathrm{~cm}^{-1}$ to $800 \mathrm{~cm}^{-1}$, which does not present a clear baseline, we have extrapolated the fit line calculated in the other region.

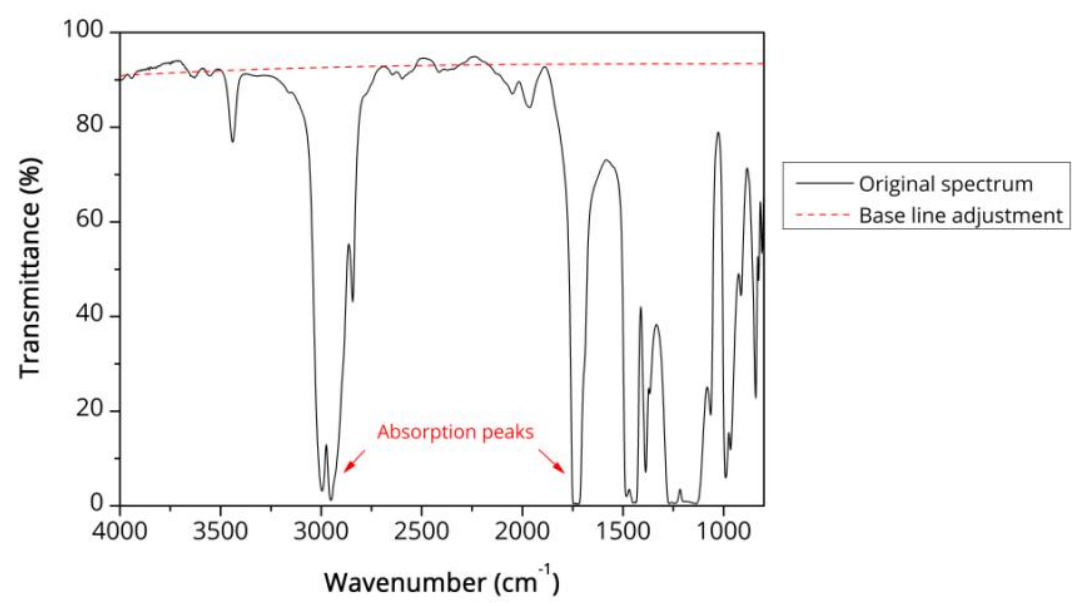

Figure 1. Example of a transmittance spectra as a function of the wavenumber of a sample of thickness $50 \pm 10 \mu \mathrm{m}$ and cell size of $14 \mathrm{~nm}$ (sample 1 in Table 1); and baseline of the spectrum after removing the absorption bands of the polymer (red dashed line).

In this work, we have calculated the scattering extinction coefficient, $K_{e, R, s c a t t}$. To do so, we have performed a fit of the base line of the transmittance spectra (as shown in the example of Figure 1) and then we have performed the analysis of equations (3) and (4). As aforementioned, $K_{e, R, s c a t t}$ accounts for the contribution of the cellular structure, so it is a good parameter to evaluate the influence of the cell size in the change from the micro- to the nanoscale. Regarding the absorption part, we could not get direct information from the experimental data, as commented previously, so the extinction coefficient we present in this paper is only the scattering part. In the last section of this work, where the total conductivity is predicted, this absorption term is calculated theoretically (see section 3.3).

One issue that might affect this calculation is the surface of the samples. The samples of this paper were prepared using a precision cutting machine, and as a result of this process they present a texture of grooves that can act as scattering surfaces, perturbating the result (i.e. increasing the scattering of the cellular materials) (see Supplementary Information, Figure S1 for images of the surface of the samples). Therefore, the scattering process in these samples has two contributions: the scattering in the cellular structure itself, but also the scattering in the surface. To factor out this second effect, that it is not of interest for our study, solid samples of different thicknesses were cut using the same method, and the scattering extinction coefficient was calculated in the same way as with the cellular materials. For the solids, as there are not any other scattering points, all the scattering is associated to the structure of the surface. This analysis leads to a value of the scattering coefficient of these solid samples, that is associated to its surface, of $33.05 \mathrm{~cm}^{-1}$. Then, this value of the scattering in the surface was subtracted to calculate the $K_{e, R, s c a t t}$ of the cellular materials to obtain the contribution of the cellular structure (see Supplementary Information, Figure S2, for the results before and after this correction). In this way, we assure that the scattering extinction coefficient presented from now on is solely due to the effect of the cellular structure. 


\subsection{Scattering mechanism: theoretical background}

As aforementioned, infrared radiation would encounter scattering phenomena when traveling through a cellular polymer due to the presence of the cells. The scattering process is strongly affected by the size of the scattering points. When the radii of the points are clearly smaller than the wavelength of the radiation, the expected scattering behavior from a theoretical perspective would be Rayleigh-like. One feature that Rayleigh scatters show is a strong dependence on the wavelength. The transmittance, $T$, depends on the wavelength, $\lambda$, as shown in equation (6), according to Rayleigh law [25], where $A$ and $B$ are constants.

$$
T=A e^{-\frac{B}{\lambda^{4}}}
$$

Another feature related to the Rayleigh scattering behavior is that the amount of radiation scattered is reduced as the size of the scattering point decreases, that is, the efficiency of the scattering reduces as size does. The scattering efficiency for a Rayleigh-like scattering behavior can be computed according to equation (7) [26]:

$$
\sigma_{\text {Ray }}=\frac{8 \pi}{3}\left(\frac{2 \pi n_{m}}{\lambda}\right)^{4} r^{6}\left(\frac{m^{2}-1}{m^{2}+2}\right)^{2}
$$

Where $n_{m}$ is the refractive index of the surrounding medium, $r$ is the radius of the scattering particle, and $m=n_{p} / n_{m}$ is the ratio of refractive indexes $\left(n_{p}\right.$ is the refractive index of the scattering particle). The scattering efficiency of one scattering particle, $Q$, is given by (9):

$$
Q=\frac{\sigma_{R a y}}{\pi r^{2}}
$$

In a general situation, the theory to describe the scattering process is the Mie theory. At low particle radii, this theory matches with the Rayleigh predictions. At large sizes of the scattering points, the Mie theory presents a limit value of the scattering efficiency equal to 2 [26].

\section{RESULTS AND DISCUSSION}

\subsection{Transmittance in the infrared region}

Figure 2 shows the transmittance in the infrared region of the samples of Table 1 with different cell sizes. The spectra presented in Figure $\mathbf{2}$ correspond to samples of thickness around $50 \pm 10 \mu \mathrm{m}$ for the sake of comparison. Note that all the samples present a similar relative density (around $\mathbf{0 . 4}$, Table $\mathbf{1}$ ). In the regions of the spectra presenting absorption bands, all the samples show full saturated bands, so there is no information about the cellular structure. However, out of these bands (for instance in the range of wavenumbers between 2750 and $2000 \mathrm{~cm}^{-1}$ ), there is a noticeable effect of the cell size. Qualitatively, we observe that the reduction of the cell size from $20 \mu \mathrm{m}$ to $14 \mathrm{~nm}$ drastically increases the transmittance, that is, the scattering of the infrared light is reduced as the cell size decreases, reaching values of transmittance close to $100 \%$ when the cell size is very small. 


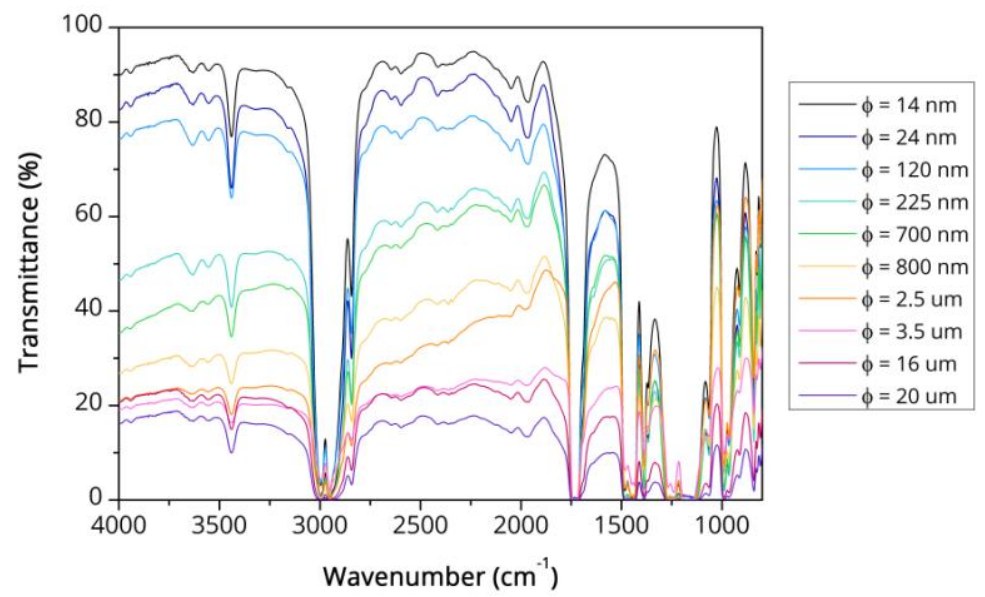

Figure 2. Transmittance as a function of the wavenumber for samples of thickness $50 \pm 10 \mu \mathrm{m}$ with variable cell size (from $14 \mathrm{~nm}$ to 20 microns).

Figure $\mathbf{3}$ shows the transmittance of the samples of Figure $\mathbf{2}$ as a function of the cell size for two fixed wavenumbers: $3300 \mathrm{~cm}^{-1}$ (3030 nm in wavelength) and $2200 \mathrm{~cm}^{-1}$ (4545 nm in wavelength). These two values were selected at regions out of the absorption bands of the PMMA to see the effect of the cellular structure. As already mentioned, it is observed that a decrease in cell size increases the transmittance dramatically.

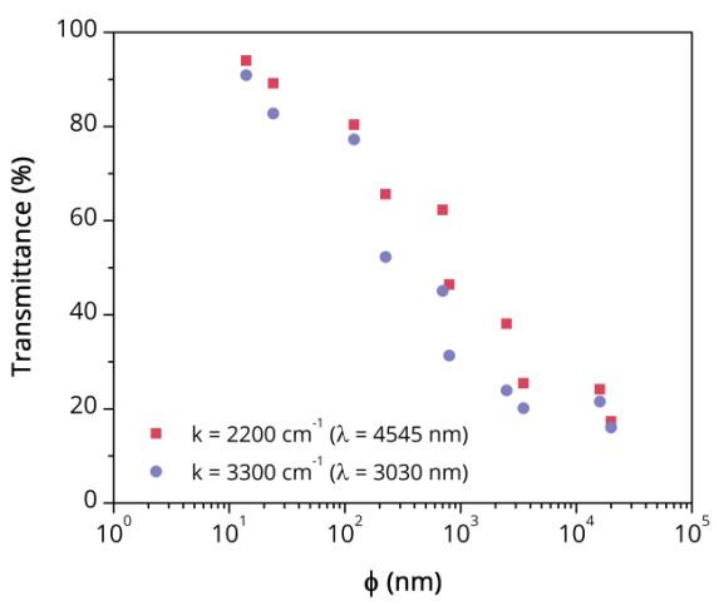

Figure 3. Transmittance as a function of the cell size for samples of thickness $50 \pm 10 \mu \mathrm{m}$ at two fixed wavelengths.

In this experiment, the infrared light used ranged from $2.5 \mu \mathrm{m}$ to $12.5 \mu \mathrm{m}$. For samples with cell sizes smaller than $1 / 10$ of these values, the expected scattering behavior from a theoretical perspective would be Rayleigh-like. As already mentioned, Rayleigh scatters show a strong dependence on the wavelength (equation (6)). To investigate this effect, the logarithm of the transmittance at specific wavelengths is plotted as a function of the parameter $1 / \lambda^{4}$ for the nanocellular samples (cell size smaller than 1 micron) in Figure 4. The wavelengths were selected in regions out of the absorption bands of the PMMA. The dependence of equation (6) is confirmed in this plot, because straight lines are obtained. 


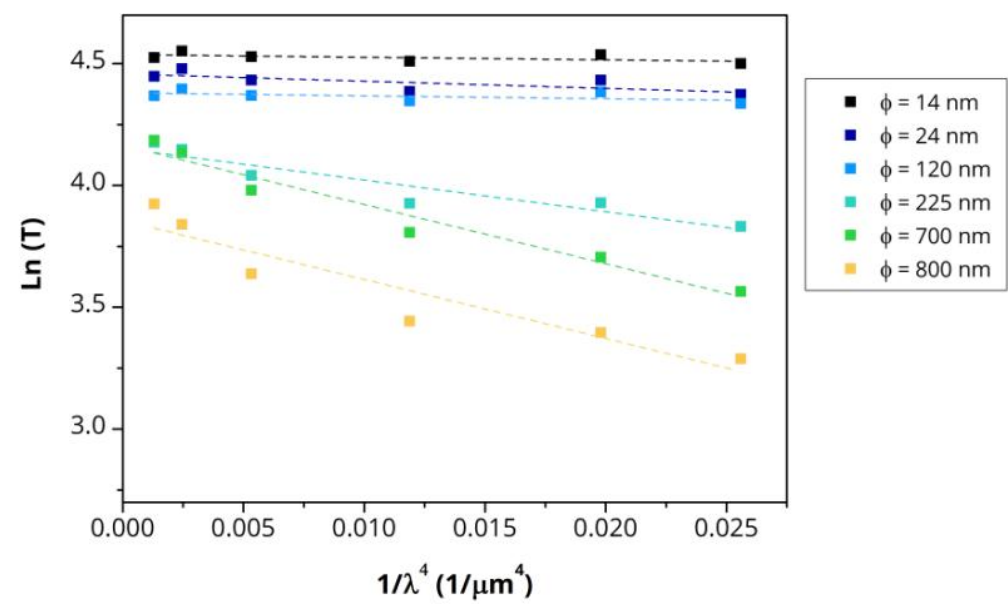

\subsection{Scattering extinction coefficient}

Using the transmittance spectra of samples with different thicknesses and performing a baseline fit, as explained previously, it is possible to calculate the extinction coefficient associated with the scattering baseline. Figure 5.a shows the Rosseland scattering extinction coefficient as a function of the cell size calculated as explained in section 2.3. This coefficient shows that there is a reduction of the extinction coefficient when the cell size is reduced to the nanoscale. At small cell sizes, the scattering extinction coefficients are very low (smaller than $\left.10 \mathrm{~cm}^{-1}\right)$, and these values start to increase as the cell size increases.

a)

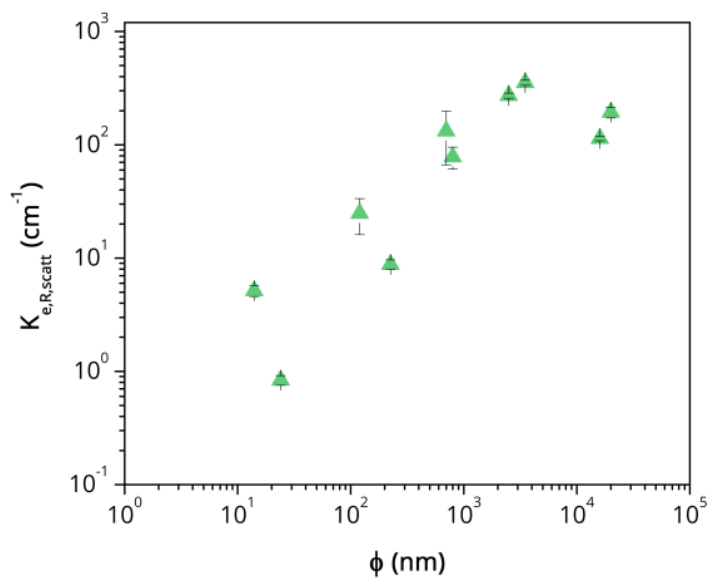

b)

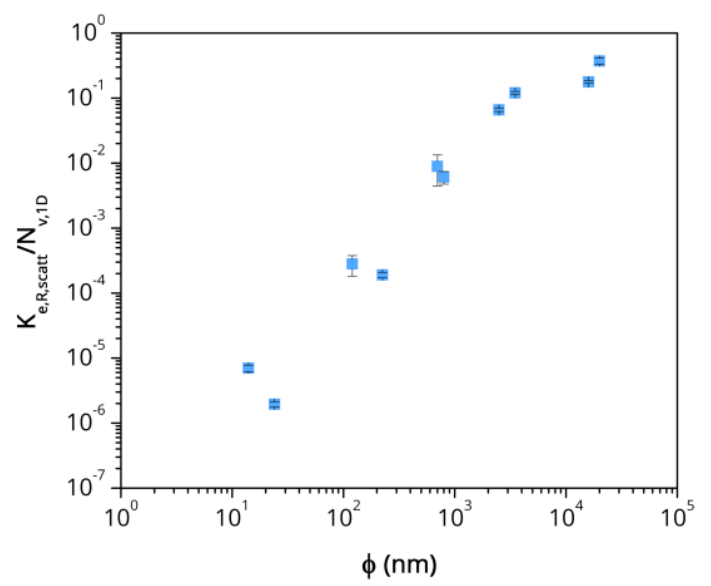

Figure 5. a) Scattering extinction coefficient as a function of the cell size, b) scattering extinction coefficient normalized by the 1D cell density (scattering efficiency of one cell) as a function of the cell size.

One interesting parameter to be calculated is the scattering efficiency of one single cell. The heat flux takes place along a given direction, and the number of cells along the sample thickness is playing a role. In a nanocellular polymer, the number of cells along the sample thickness is much higher than in a microcellular polymer for the same density. That is, the number of scattering points is higher in nanocellular polymers. Then, one way to normalize the 
$K_{e, R, s c a t t}$ is considering the linear cell density, that is, the number of cells per unit of length.

257 From the standard definition of the cell density [27], the linear cell density $N_{v, 1 D}$ can be

258 calculated theoretically as shown in equation (9), where $\phi$ is the cell size and $\rho_{r}$ is the relative 259 density. Note that the units of $N_{v, 1 D}$ are $\mathrm{cm}^{-1}$, so when $K_{e, R, s c a t t}$ is normalized by this value the 260 result is unitless.

261

262

263

264

265

266

267

268

269

270

271

272

273

274

275

276

277

278

279

280

281

282

283

284

285

286

287

288

289

$$
N_{v, 1 D}=\left[\frac{6}{\pi \phi^{3}}\left(1-\rho_{r}\right)\right]^{1 / 3}
$$

Figure 5.b shows the result of dividing $K_{e, R, s c a t t}$ by the linear cell density calculated as defined in (9). Note that the parameter $K_{e, R, s c a t t} / N_{v, 1 D}$ shows a much clear dependence with the cell size because of the fact that the cell density differences are corrected. Increasing the cell size causes an increment of the scattering efficiency, or in other words, the reduction of the cell size to the nanoscale reduces the efficiency of the cells as scattering centers. Note that the cell density correction also corrects the small density variations among samples (see Table 1), since the cell density is calculated based both on the cell size and the relative density. For this reason, the parameter $K_{e, R, s c a t t} / N_{v, 1 D}$ presented in Figure 5.b, that accounts for the scattering effect of one single cell, is a general result that could be applied to any cellular material with this range of cell sizes independently on the density.

The trend observed in Figure 5.b is similar to the expected theoretical behavior for Rayleigh scatters, that is, the efficiency of the scattering reduces as size does (equation (8)). To correlate these results with the theoretical predictions, Figure 6 shows the predicted theoretical scattering efficiency of Rayleigh particles as a function of the unitless parameter $x=2 \pi n_{m} r / \lambda$ (size parameter). The efficiency of the scattering increases as the particle radius increases. When the particle size is very large (that is, $x \gg 1$ ), the scattering behavior would move to the Mie regime, reaching a limit value of $Q$ equal 2 [26]. In Figure 6 we have included the values of $K_{e, R, s c a t t} / N_{v, 1 D}$, what we have called "scattering efficiency of one cell", calculated at the two limit wavelengths, 2500 and $12500 \mathrm{~nm}$. Data from a PU foam with a much higher cell size (366 um) was also included for comparison [28]. These experimental values show a similar trend in comparison with the theoretical predictions. The scattering efficiency increases linearly as cell sizes does, reaching the Mie limit for large cell sizes. Figure 6 proves that nanocellular polymers present also Rayleigh scattering with regard to the cell size dependence.

Therefore, the results of this work prove that the transmittance in the infrared region of nanocellular polymers follows the theoretical trends in terms of wavelength and cell size dependence. The values estimated in this paper give a reasonable estimation of the behavior of infrared radiation interacting with nanocellular polymers. 


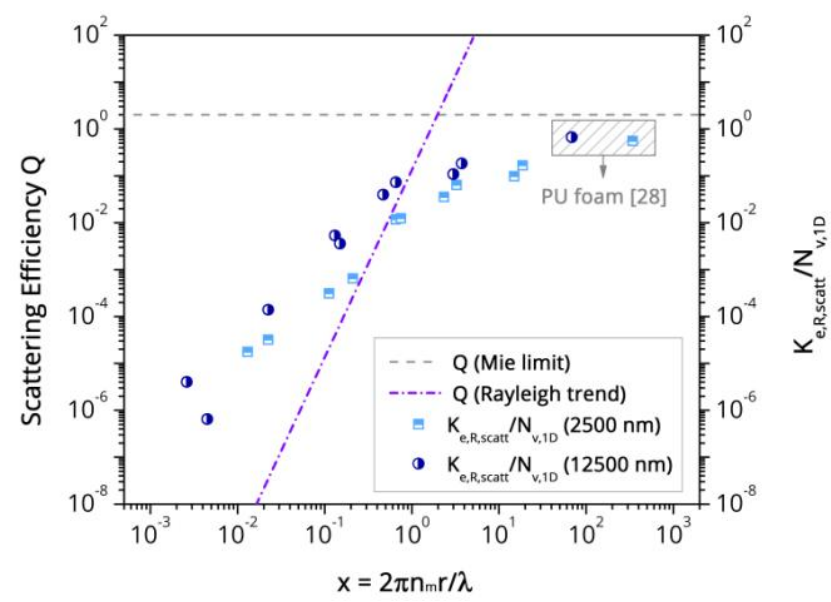

Figure 6. Right axis: Scattering efficiency $Q$ for a Rayleigh-like behavior and Mie limit as a function of the size parameter $x$. Left axis: Scattering extinction coefficient normalized by the 1D cell density as a function of the size parameter $x$.

\subsection{Modeling the radiation contribution}

Once the extinction coefficient is calculated, it is possible to use these experimental values to predict the radiation contribution and model the thermal conductivity. Recall from equation (5) that we split the extinction coefficient into two parts. The absorption contribution, $K_{e, R, a b s}$, can be modelled using the extinction coefficient of the solid polymer, $K_{P}$, and the relative density, as shown in equation (10), since the amount of radiation absorbed will be proportional to the amount of solid polymer [29]:

$$
K_{e, R, a b s}=K_{P} \rho_{r}
$$

To quantify the scattering term, the data of Figure 5.b corresponding to the scattering efficiency of one cell was adjusted to a potential equation of the form:

$$
K_{e, R, s c a t t} / N_{v, 1 D}=A \phi^{B}
$$

Where $A$ and $B$ are dimensionless experimental parameters. In particular, for our data, $A$ is $5.02 \cdot 10^{-8}$ and $B$ is 1.68 . Therefore, the total extinction coefficient can be expressed as:

$$
K_{e, R}=K_{e, R, a b s}+K_{e, R, s c a t t}=K_{P} \rho_{r}+N_{v, 1 D} A \phi^{B}
$$

Where $N_{v, 1 D}$ is calculated from the density and the cell size according to equation (9). The radiation term can be then calculated as (2) by using this semi-empirical extinction coefficient defined in (12).

In aerogels, it is claimed that the extinction of the infrared radiation is only due to absorption and not scattering due to the small pore size [13]. To see if this assumption can also be made in nanocellular polymers, it is interesting to calculate the relative contribution of the scattering and the absorption part to the total extinction coefficient for a low density material (relative density 0.05 ) (Figure 7). One key parameter in this study is the extinction coefficient of the solid polymer, $K_{P}$. Unfortunately, and as far as the authors know, there are not data about this parameter for PMMA in this wavelength range. To do some first estimations, two extreme values have been selected for the graphs of Figure 7. First, a low value of $K_{P} 140 \mathrm{~cm}^{-1}$ measured for low-density polyethylene [17], and then a high value of $600 \mathrm{~cm}^{-1}$ calculated for 
polyurethane foams [5]. It is observed that, in both cases, the contribution of the scattering is reduced as the cell size reduces. For very small cell sizes, it might be a good approximation to neglect the scattering part, especially if the absorption of the solid is high (Figure 7.b). However, as cell size increases, the contribution of the scattering becomes more relevant. For cell sizes of 200-300 nm, that are typical values reported in many works in the literature, the contribution can be higher than $75 \%$ (for $K_{P}=140 \mathrm{~cm}^{-1}$, Figure 7.a) or at least higher than $40 \%$ (for $K_{P}=600 \mathrm{~cm}^{-1}$, Figure 7.b). Thus, at these cell sizes, we cannot neglect this contribution, and to model properly the thermal conductivity both aspects, absorption and scattering, must be included in the equations.

a)

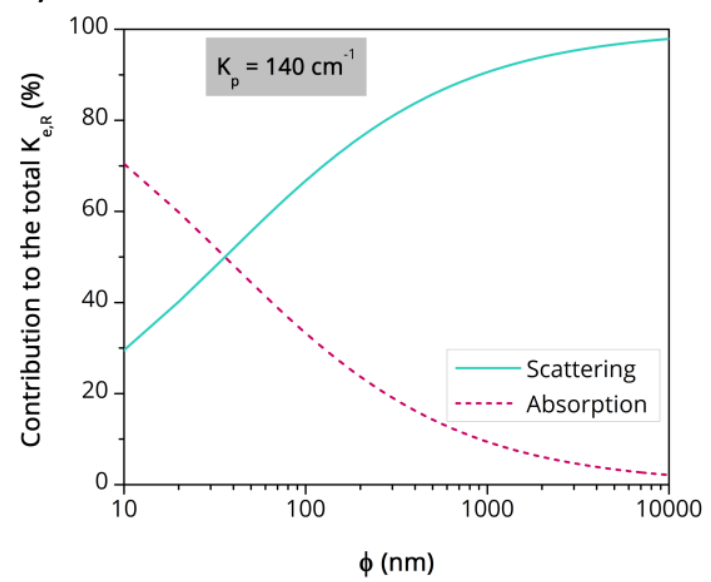

b)

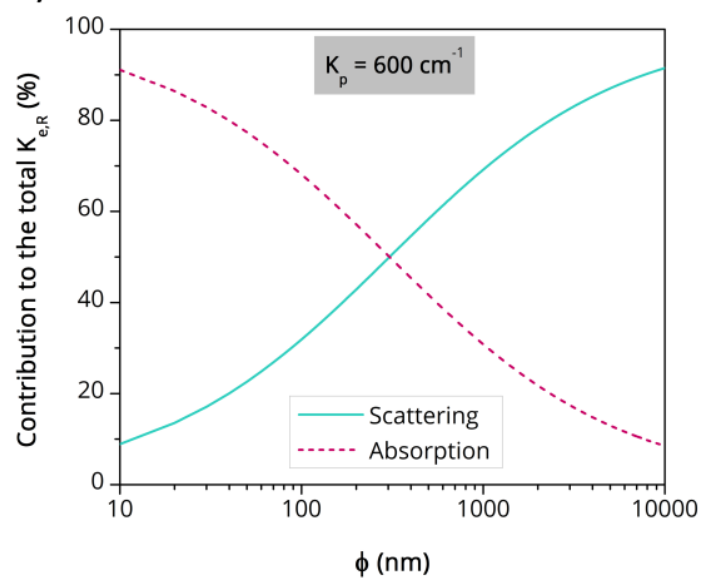

Figure 7. Contribution to the total extinction coefficient of the scattering and the absorption parts for a low relative density material (relative density $=0.05$ ), for two different extinction coefficients of the solid: a) $K_{P}=140 \mathrm{~cm}^{-1}$ and b) $K_{P}=600 \mathrm{~cm}^{-1}$.

Regarding the other contributions to calculate the thermal conductivity in equation (1), the convection term is known to be negligible for cell sizes smaller than $2 \mathrm{~mm}[5,24,30]$. For nanocellular polymers, the conduction terms, $\lambda_{s}$ and $\lambda_{g}$, are usually described by the following equations [31-33]:

$$
\begin{gathered}
\lambda_{s}=g \lambda_{s}^{\prime} \rho_{r} \\
\lambda_{g}=\frac{\lambda_{g 0}^{\prime}}{1+\frac{2 \beta l_{g}}{\phi}}\left(1-\rho_{r}\right)
\end{gathered}
$$

Where $\lambda_{s}^{\prime}$ is the thermal conductivity of the solid polymer, and $g$ is a an efficiency-structural factor proposed by Glicksman [5]. This $g$ factor ranges from $1 / 3$ to 1 . For medium-high density materials it usually takes values close to $1[34,35]$, while this factor is $2 / 3$ for closed-cell low density materials and it reaches the minimum value of $1 / 3$ for materials with a high fraction of the solid phase in the struts (i.e., open cell structures) [5]. Regarding the gas phase, $\lambda_{g 0}^{\prime}$ is the thermal conductivity of the gas in the cells $(26 \mathrm{~mW} / \mathrm{mK}$ for air at atmospheric pressure and room temperature), $\beta$ is a factor correlating the energy transfer between gas molecules and the structure [33] (1.64 for air [32]) and $l_{g}$ is the mean free path of the gas molecules $\left(l_{g} \approx 70\right.$ $n m$ for air $[35,36])$.

The thermal conductivity of nanocellular polymers was calculated using equations (1), (2), (12), (13), and (14). Thermal conductivity of PMMA is $212 \mathrm{~mW} / \mathrm{mK}$ [37], and as in the previous example, two extreme extinction coefficient of the solid $\left(K_{P}\right)$ have been used: 140 and $600 \mathrm{~cm}^{-}$ 
$347{ }^{1}$. Regarding the structural factor $g$, a value of $2 / 3$ was selected, since the predictions were 348 mainly focused in the low-density region $\left(\rho_{r} \leq 0.2\right)$. Figure 8 shows the predicted thermal 349 conductivity as a function of the cell size for a fixed relative density of 0.05 (Figure 8.a), and as 350 a function the relative density for a fixed cell size of $50 \mathrm{~nm}$ (Figure 8.b). The predictions 351 without including the radiation term are also plotted in this graph for the sake of comparison. 352 The relative contribution of the radiation mechanism for the two values of $K_{P}$ are presented in 353 Figure 8.c and Figure 8.d. Note that in these predictions, some critical assumptions have been 354 made: the cellular structure was considered monomodal (there might be an influence of the 355 cell size distribution [37]), homogeneous, 100\% closed cell, etc. Thus, these predictions could 356 differ slightly from real conductivity values, but they show qualitatively the expected trends.

357 It is observed that there is a significant difference between the models presented in this work 358 and the predictions without including the radiation term. Also, the predictions are very 359 sensitive to the value of $K_{P}$. For a low $K_{P}$ and relative density of 0.05 , a minimum conductivity 360 appears at a cell size of around $70 \mathrm{~nm}$, but this minimum is not observed with a higher $K_{P}$ 361 (Figure 8.a). In the worse situation (lowest $K_{P}$ ), for a relative density of 0.05 , the minimum conductivity calculated is around $23 \mathrm{~mW} / \mathrm{mK}$. The contribution of the radiation term to the total thermal conductivity increases as cell size decreases, ranging the maximum values from 40 to $70 \%$ depending on $K_{P}$ (Figure 8.c).

365 Regarding the density dependency (Figure 8.b), the conductivity reduces with density up to a minimum. For $50 \mathrm{~nm}$, the minimum conductivity is $15.5 \mathrm{~mW} / \mathrm{mK}$ for a density of 0.032 for the high $K_{P}$, whereas the value is $22.7 \mathrm{~mW} / \mathrm{mK}$ for a density of 0.034 for $K_{P}=140 \mathrm{~cm}^{-1}$. The contribution of the radiation term to the total thermal conductivity increases as density decreases, ranging the maximum values from 40 to $80 \%$ depending on $K_{P}$ (Figure 8.d). 
a)

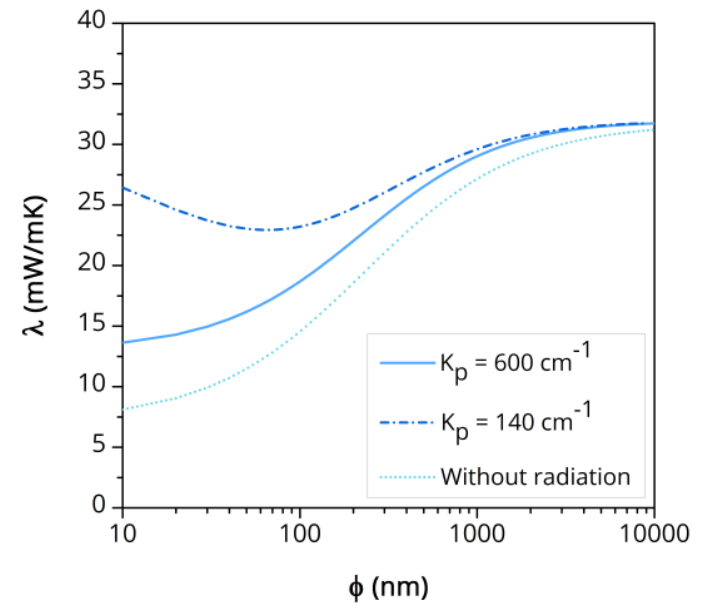

b)

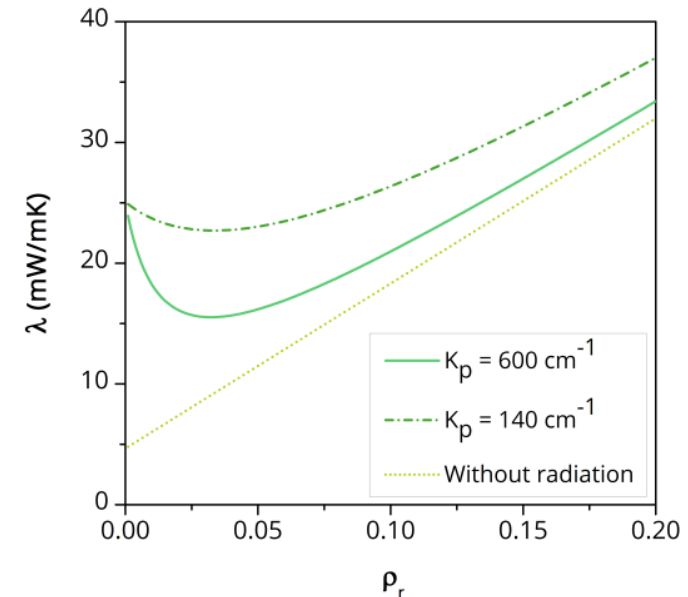

c)

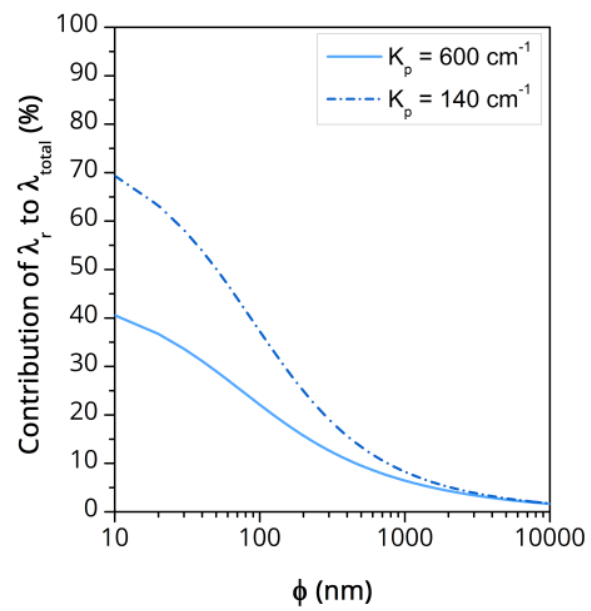

d)

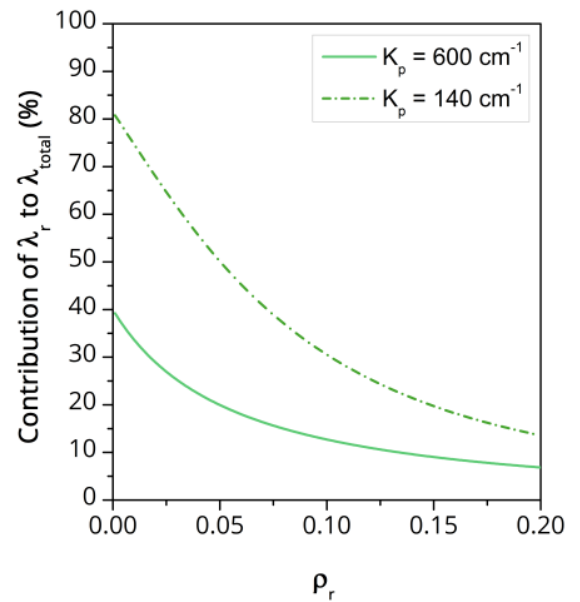

373

Figure 8. a) Theoretical thermal conductivity of nanocellular polymers based on PMMA according to the model including the radiation term and without it and for two different extinction coefficients of the solid a) as a function of the cell size for relative density of 0.05 , and b) as a function of the relative density for cell size of $50 \mathrm{~nm}$. Contribution of the radiation mechanism to the total thermal conductivity: b) for a relative density of 0.05 as a function of the cell size and d) for a cell size of $50 \mathrm{~nm}$ as a function of the relative density.

The great potential of nanocellular polymers as excellent thermal insulators has been pointed out in various works $[35,38,39]$. The results of this work do not contradict these claims. Our predictions show that even though the radiation term is included, it would be possible to obtain low thermal conductivities with nanocellular polymers, for instance, for a relative density of 0.05 and a cell size of $70 \mathrm{~nm}$ the predicted thermal conductivity would be as low as $23 \mathrm{~mW} / \mathrm{mK}$. However, the most optimistic predictions of the early years of the field of nanocellular polymers (reductions of 2.5 times $[35,40]$ ) seem now almost impossible to reach using homogeneous polymeric systems once the radiation contribution has been understood and experimentally characterized.

Further work would be needed to understand how to reduce this contribution in nanocellular polymers. The use of bimodal structures, with micron-sized pores able to scatter radiation and also helping to reduce the density, might be beneficial in this sense [37]. Also, IR nanometric opacifiers could be included to reduce the $K_{P}$ of the solid matrix and decrease the radiation 
contribution[41]. The results of this work would pave the way for future developments to further exploit the exciting properties of nanocellular polymers.

\section{CONCLUSIONS}

Transmittance spectra in the infrared region of a collection of micro- and nanocellular polymers were measured to investigate the interaction of nanocellular structures with infrared radiation. A collection of PMMA-based micro- and nanocellular polymers with a constant density and a wide range of cell sizes (from $14 \mathrm{~nm}$ to $20 \mu \mathrm{m}$ ) were used for these measurements. Results show that there is strong increase of the transmittance out of the absorption bands as the cell size reduces to the nanoscale. The dependencies of the transmittance with both the wavelength and the cell size prove that nanometric cells act as Rayleigh scattering particles.

By performing a fit of the scattering baseline, it is possible to use the transmittance spectra to calculate the scattering extinction coefficient of nanocellular polymers. The results of this paper show that the extinction coefficient reduces as the cell size decreases. The values were corrected by the linear cell density to calculate the scattering efficiency of one cell as a function of the cell size.

From these values, it is possible to calculate the total extinction coefficient and to weight the relevance of the scattering part. We conclude that the scattering can be neglected for very low cell sizes (smaller than $200 \mathrm{~nm}$ ), but it must be considered for larger cell sizes. The thermal conductivity of nanocellular polymers was modeled including the radiation contribution, showing that at low relative densities and small cell sizes this term becomes significant in nanocellular polymers.

\section{Acknowledgments}

Financial assistance from MINECO, FEDER, UE (MAT2015-69234-R), the Junta de Castile and Leon (VA275P18) and Spanish Ministry of Science, Innovation and Universities (RTI2018098749-B-100) are gratefully acknowledged. Financial assistance from EREN (Ente Regional de la Energía de Castilla y León EREN_2019_L4_UVA) is gratefully acknowledged. This project has received funding from the European Union's Horizon 2020 research and innovation programme under grant agreement No 730872.

\section{REFERENCES}

[1] International Energy Agency (IEA), Technology Roadmap. Energy efficient building envelopes, (2013). doi:10.1007/SpringerReference_7300.

[2] B.P. Jelle, Traditional, state-of-the-art and future thermal building insulation materials and solutions - Properties, requirements and possibilities, Energy Build. 43 (2011) 2549-2563. doi:10.1016/j.enbuild.2011.05.015.

[3] L. Aditya, T.M.I. Mahlia, B. Rismanchi, H.M. Ng, M.H. Hasan, H.S.C. Metselaar, O. Muraza, H.B. Aditiya, A review on insulation materials for energy conservation in buildings, Renew. Sustain. Energy Rev. 73 (2017) 1352-1365. 
436 [4] D. Eaves, Handbook of Polymer Foams, Rapra Technology, United Kingdom, 2004.

437 [5] N.C. Hilyard, A. Cunningham, Low density cellular plastics--Physical basis of behaviour, Chapman and Hall, London, 1994.

[6] R.J.J. Williams, C.M. Aldao, Thermal conductivity of plastic foams, Polym. Eng. Sci. 23

[7] L. Glicksman, M. Schuetz, M. Sinofsky, Radiation heat transfer in foam insulation, Int. J.

[8] S. Perez-Tamarit, B. Notario, E. Solorzano, M.A. Rodriguez-Perez, Light transmission in nanocellular polymers: are semi-transparent cellular polymers possible?, Mater. Lett.

[9] J. Martin-de Leon, V. Bernardo, M.A. Rodriguez-Perez, Key Production Parameters to Obtain Transparent Nanocellular PMMA, Macromol. Mater. Eng. 1700343 (2017) 1-5. doi:10.1002/mame.201700343.

[10] J. Martín-de León, J.L. Pura, V. Bernardo, M.Á. Rodríguez-Pérez, Transparent nanocellular PMMA: Characterization and modeling of the optical properties, Polymer (Guildf). 170 (2019) 16-23. doi:10.1016/j.polymer.2019.03.010.

[11] R. Baetens, B. Petter, A. Gustavsen, Aerogel insulation for building applications : A state-of-the-art review, Energy Build. 43 (2011) 761-769. doi:10.1016/j.enbuild.2010.12.012.

[12] L.W. Hrubesh, R.W. Pekala, Thermal properties of organic and inorganic aerogels, J. Mater. Res. 9 (1994) 731-738. doi:10.1557/JMR.1994.0731.

[13] U. Heinemann, R. Caps, J. Fricke, Radiation-conduction interaction : An investigation on silica aerogels, Int. J. Heat Mass Transf. 39 (1996) 2115-2130. doi:10.1016/00179310(95)00313-4.

[14] P. Ferkl, R. Pokorný, M. Bobák, J. Kosek, Heat transfer in one-dimensional micro- and nano-cellular foams, Chem. Eng. Sci. 97 (2013) 50-58. doi:10.1016/j.ces.2013.04.018.

[15] G. Wang, C. Wang, J. Zhao, G. Wang, C.B. Park, G. Zhao, Modelling of thermal transport through a nanocellular polymer foam: Toward the generation of a new superinsulating material, Nanoscale. 9 (2017) 5996-6009. doi:10.1039/c7nr00327g.

[16] L.R. Glicksman, Heat transfer in foams, in: N.C. Hilyard, A. Cunningham (Eds.), Low Density Cell. Plast., Springer, Dordrecht, 1994: pp. 104-152. doi:10.1007/978-94-0111256-7 5.

[17] R.A. Campo-Arnáiz, M.A. Rodríguez-Pérez, B. Calvo, J.A. De Saja, Extinction coefficient of polyolefin foams, J. Polym. Sci. Part B Polym. Phys. 43 (2005) 1608-1617. doi:10.1002/polb.20435.

[18] O.A. Almanza, J.A. de Saja, M.A. Rodriguez-Perez, Prediction of the Radiation Term in the Thermal Conductivity of Crosslinked Closed Cell Polyolefin Foams, J. Polym. Sci. Part B Polym. Phys. 38 (2000) 993-1004. doi:10.1002/(SICI)10990488(20000401)38:7<993::AID-POLB10>3.0.CO;2-J.

[19] V. Bernardo, J. Martin-de Leon, J. Pinto, T. Catelani, A. Athanassiou, M.A. RodriguezPerez, Low-density PMMA/MAM nanocellular polymers using low MAM contents: 
Production and characterization, Polymer (Guildf). 163 (2019) 115-124. doi:10.1016/j.polymer.2018.12.057.

[20] J. Pinto, E. Solórzano, M.A. Rodriguez-Perez, J.A. De Saja, Characterization of the cellular structure based on user-interactive image analysis procedures, J. Cell. Plast. 49 (2013) 555-575. doi:10.1177/0021955X13503847.

[21] J. Martin de-Leon, V. Bernardo, M.A. Rodriguez-Perez, Low Density Nanocellular Polymers Based on PMMA Produced by Gas Dissolution Foaming: Fabrication and Cellular Structure Characterization, Polymers (Basel). 8 (2016) 1-16. doi:10.3390/polym8070265.

[22] V. Bernardo, J. Martin-de Leon, E. Laguna-Gutierrez, T. Catelani, J. Pinto, A. Athanassiou, M.A. Rodriguez-Perez, Understanding the role of MAM molecular weight on the production of PMMA/MAM nanocellular polymers, Polymer (Guildf). 153 (2018) 262-270. doi:10.1016/j.polymer.2018.08.022.

[23] V. Bernardo, F. Van Loock, J. Martin-de Leon, N.A. Fleck, M.A. Rodriguez-Perez, Mechanical Properties of PMMA-Sepiolite Nanocellular Materials with a Bimodal Cellular Structure, Macromol. Mater. Eng. 1900041 (2019) 1-12. doi:10.1002/mame.201900041.

[24] L.J. Gibson, M. Ashby, Cellular solids: structure and properties, 2nd Editio, Cambridge University Press, 1997.

[25] Wanqing Cao, A.J. Hunt, Improving the visible transparency of silica aerogels, J. Non. Cryst. Solids. 176 (1994) 18-25. doi:10.1016/0022-3093(94)90206-2.

[26] A.J. Cox, A.J. DeWeerd, J. Linden, An experiment to measure Mie and Rayleigh total scattering cross sections, Am. J. Phys. 70 (2002) 620-625. doi:10.1119/1.1466815.

[27] V. Kumar, N.P. Suh, A process for making microcellular parts, Polym. Eng. Sci. 30 (1990) 1323-1329. doi:https://doi.org/10.1002/pen.760302010.

[28] M. Santiago-Calvo, J. Tirado-Mediavilla, J.C. Rauhe, L.R. Jensen, J.L. Ruiz-Herrero, F. Villafañe, M.Á. Rodríguez-Pérez, Evaluation of the thermal conductivity and mechanical properties of water blown polyurethane rigid foams reinforced with carbon nanofibers, Eur. Polym. J. 108 (2018) 98-106. doi:10.1016/j.eurpolymj.2018.08.051.

[29] M.E. Fajardo, C.H. Neel, D.G. Lacina, Using mid-infrared external reflectance spectroscopy to distinguish between different commercially produced poly[methyl methacrylate] (PMMA) samples - A null result, AIP Conf. Proc. 1979 (2018). doi:10.1063/1.5044948.

[30] M. Alvarez-Lainez, M.A. Rodríguez-Pérez, J.A. de Saja, Thermal Conductivity of OpenCell Polyolefin Foams, J. Polym. Sci. Part B Polym. Phys. 46 (2008) 212-221. doi:10.1002/polb.

[31] P.G. Collishaw, J.R.G. Evans, An Assessment of Expressions for the Apparent Thermal Conductivity of Cellular Materials, J. Mater. Sci. 29 (1994) 2261-2273. doi:10.1007/BF00363413.

[32] S. Song, M.M. Yovanovich, F.O. Goodman, Thermal Gap Conductance of Conforming Surfaces in Contact, J. Heat Transfer. 115 (1993) 533-540. doi:10.1115/1.2910719.

[33] Z. Li, C. Zhu, X. Zhao, A theoretical and numerical study on the gas-contributed thermal conductivity in aerogel, Int. J. Heat Mass Transf. 108 (2017) 1982-1990. 
[34] M. Saadatfar, C.H. Arns, M.A. Knackstedt, T. Senden, Mechanical and transport properties of polymeric foams derived from 3D images, Colloids Surfaces A Physicochem. Eng. Asp. 263 (2005) 284-289. doi:10.1016/j.colsurfa.2004.12.040.

[35] B. Notario, J. Pinto, E. Solorzano, J.A. de Saja, M. Dumon, M.A. Rodriguez-Perez, Experimental validation of the Knudsen effect in nanocellular polymeric foams, Polymer (Guildf). 56 (2015) 57-67. doi:10.1016/j.polymer.2014.10.006.

[36] C. Forest, P. Chaumont, P. Cassagnau, B. Swoboda, P. Sonntag, Polymer nano-foams for insulating applications prepared from CO 2 foaming, Prog. Polym. Sci. 41 (2015) 122145. doi:10.1016/j.progpolymsci.2014.07.001.

[37] V. Bernardo, J. Martin-de Leon, J. Pinto, R. Verdejo, M.A. Rodriguez-Perez, Modeling the heat transfer by conduction of nanocellular polymers with bimodal cellular structures, Polymer (Guildf). 160 (2019) 126-137. doi:10.1016/j.polymer.2018.11.047.

[38] G. Wang, J. Zhao, L.H. Mark, G. Wang, K. Yu, C. Wang, C.B. Park, G. Zhao, Ultra-tough and super thermal-insulation nanocellular PMMA/TPU, Chem. Eng. J. 325 (2017) 632646. doi:10.1016/j.cej.2017.05.116.

[39] S. Liu, J. Duvigneau, G.J. Vancso, Nanocellular polymer foams as promising high performance thermal insulation materials, Eur. Polym. J. 65 (2015) 33-45. doi:10.1016/j.eurpolymj.2015.01.039.

[40] S. Costeux, CO2-blown nanocellular foams, J. Appl. Polym. Sci. 131 (2014) 41293(1)41293(16). doi:10.1002/app.41293.

[41] M. Arduini, J. Manara, C. Vo, Modeling of radiative properties of polystyrene foams containing IR-opacifiers, Cell. Polym. 35 (2016) 49-66. 


\section{Credit Author Statement}

Victoria Bernardo: Investigation, Formal analysis, Writing - Original Draft; Judith Martin-de Leon: Investigation, Writing - Review and Editing; Javier Pinto: Investigation, Writing - Review and Editing; Ulrich Schade: Methodology: Writing - Review and Editing: Miguel Angel Rodriguez-Perez: Conceptualization, Supervision, Writing - Review and Editing 


\section{${ }^{*}$ Declaration of Interest Statement}

\section{Declaration of interests}

$\bigotimes$ The authors declare that they have no known competing financial interests or personal relationships that could have appeared to influence the work reported in this paper.

$\square$ The authors declare the following financial interests/personal relationships which may be considered as potential competing interests:

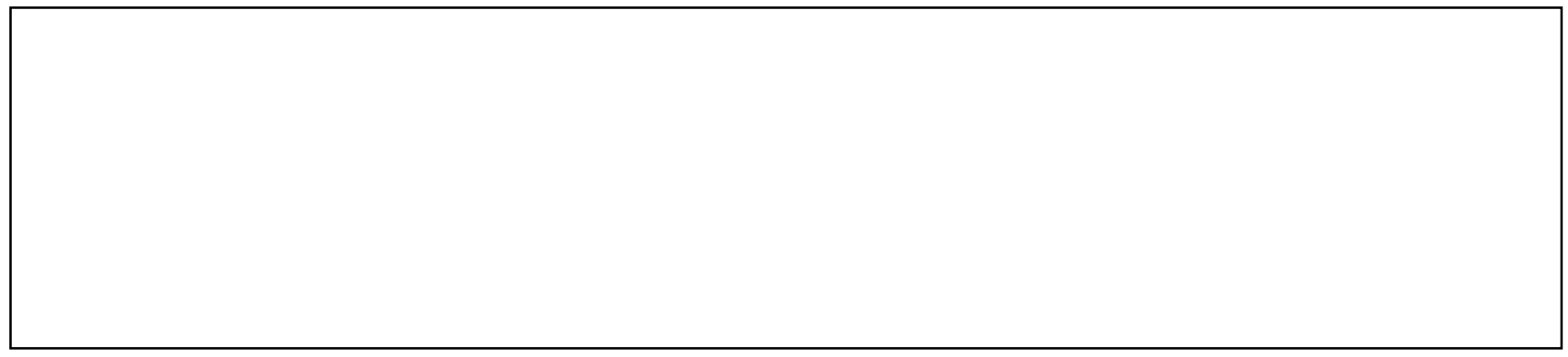

